\title{
Regulation of Axonal Midline Guidance by Prolyl 4-Hydroxylation in Caenorhabditis elegans
}

\author{
Nanna Torpe and Roger Pocock \\ Biotech Research and Innovation Centre, University of Copenhagen, 2200 Copenhagen, Denmark
}

Neuronal wiring during development requires that the growth cones of axons and dendrites are correctly guided to their appropriate targets. As in other animals, axon growth cones in Caenorhabditis elegans integrate information in their extracellular environment via interactions among transiently expressed cell surface receptors, their ligands, and the extracellular matrix (ECM). Components of the ECM undergo a wide variety of post-translational modifications that may affect efficacy of binding to neuronal guidance molecules. The most common modification of the ECM is prolyl 4-hydroxylation. However, little is known of its importance in the control of axon guidance. In a screen of prolyl 4-hydroxylase (P4H) mutants, we found that genetic removal of a specific P4H subunit, DPY-18, causes dramatic defects in C. elegans neuroanatomy. In $d p y$ - 18 mutant animals, the axons of specific ventral nerve cord neurons do not respect the ventral midline boundary and cross over to the contralateral axon fascicle. We found that these defects are independent of the known role of $d p y$-18 in regulating body size and that $d p y-18$ acts from multiple tissues to regulate axon guidance. Finally, we found that the neuronal defects in $d p y$-18 mutant animals are dependent on the expression of muscle-derived basement membrane collagens and motor neuron-derived ephrin ligands. Loss of $d p y-18$ causes dysregulated ephrin expression and this is at least partially responsible for the neurodevelopmental defects observed. Together, our data suggest that DPY-18 regulates ephrin expression to direct axon guidance, a role for P4Hs that may be conserved in higher organisms.

Key words: axon guidance; collagen; ephrin

\section{Introduction}

Faithful development of the nervous system requires that neurons assimilate guidance information supplied from other cells and the extracellular environment (Tessier-Lavigne and Goodman, 1996; Yu and Bargmann, 2001). Such guidance molecules are sensed and processed by neurons to facilitate accurate targeting of axonal and dendritic projections. The ventral nerve cord (VNC) of Caenorhabditis elegans contains many neurons that project along the length of the animal (White et al., 1976). The guidance of these neurons is regulated by multiple conserved molecular pathways and is also sensitive to environmental perturbations (Zallen et al., 1998; Hobert and Bülow, 2003; Bülow et al., 2004; Rhiner et al., 2005; Boulin et al., 2006; Pocock and Hobert, 2008; Steimel et al., 2010).

Basement membranes (BMs) are specialized areas of extracellular matrix (ECM) that act as integration centers for cell-

Received April 2, 2014; revised Oct. 7, 2014; accepted Oct. 21, 2014.

Author contributions: N.T. and R.P. designed research; N.T. and R.P. performed research; N.T. and R.P. analyzed data; N.T. and R.P. wrote the paper.

This work was supported by grants from the European Research Council (ERC Starting Grant number 260807 to R.P.) and the Lundbeck Foundation (project number R67-A6094 to R.P). We thank members of the Pocock Laboratory for comments on the manuscript, Dave Hall for electron microscopy expertise, and Agnieszka Podolska for help with qRT-PCR experiments. Some strains were provided by the Caenorhabditis Genetics Center (University of Minnesota), which is funded by the National Institutes of Health Office of Research Infrastructure Programs (P40 0D010440), and by Shohei Mitani at the National Bioresource Project (Japan).

The authors declare no competing financial interests.

Correspondence should be addressed to Roger Pocock at the above address. E-mail: roger.pocock@bric.ku.dk. DOI:10.1523/JNEUROSCI.1322-14.2014

Copyright $\odot 2014$ the authors $\quad 0270-6474 / 14 / 3316348-10 \$ 15.00 / 0$ surface and extracellular-released guidance molecules (VarelaEchavarría and Guthrie, 1997). This environment provides a complex arena for axonal growth cones to precisely map their position in time and space to enable correct axon guidance. A major structural and signaling constituent of the BM is collagen (Paulsson, 1992) and functions of collagen in the control of axon guidance has been reported in a number of systems. Collagen XVIII is required for motor neuron axon guidance in both $C$. elegans and zebrafish (Ackley et al., 2001; Schneider and Granato, 2006), and collagen XIXa1 regulates motor axon guidance in zebrafish (Hilario et al., 2010). Central in the control of collagen biosynthesis are the collagen prolyl 4-hydroxylase enzymes (P4Hs), which hydroxylate multiple proline residues in collagen (Myllyharju, 2003). This modification is required for collagen triple helix formation and stability. To date, however, the importance of prolyl 4-hydroxylation in the control of neuronal development is not well understood.

In C. elegans, four genes encode collagen P4Hs: $d p y$-18 (also known as phy-1), phy-2, phy-3, and phy-4 (Friedman et al., 2000; Hill et al., 2000; Keskiaho et al., 2008). These genes control embryonic development, body size, and morphogenesis (Friedman et al., 2000; Hill et al., 2000). Previous work described a role for the HIF-1-prolyl hydroxylase EGL-9 in axon guidance regulation (Pocock and Hobert, 2008); however, there is no known role for the collagen $\mathrm{P} 4 \mathrm{H}$ enzymes in neuronal development. In this study, we found that loss of $d p y-18$ causes defects in hermaphrodite-specific neuron (HSN) development, in addition to a subset of other VNC neurons in C. elegans. Genetic analysis re- 
A
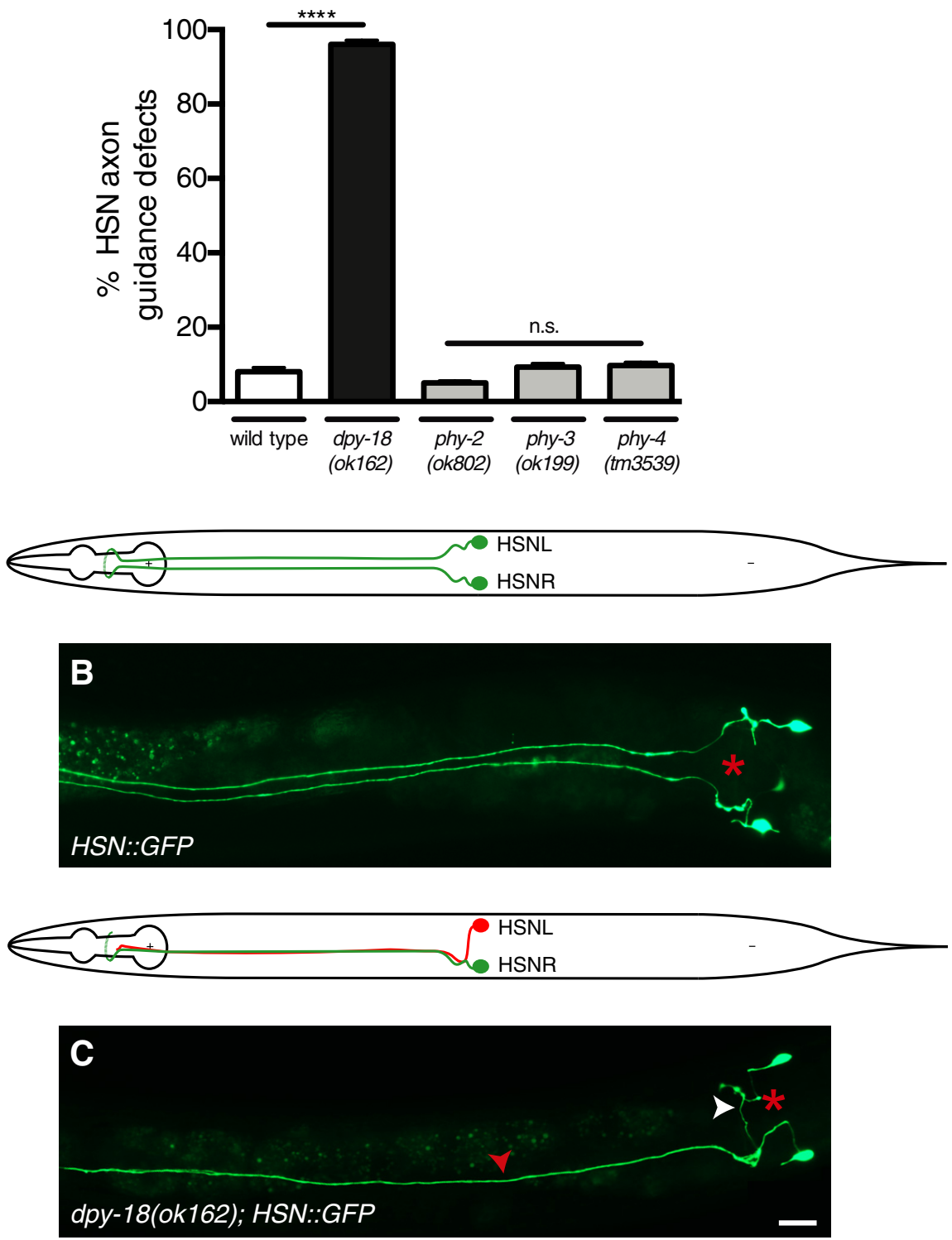

Figure 1. $\quad d p y-18$ is required for HSNL axon guidance. A, Quantification of HSN axonal cross-over defects in collagen P4H mutants. HSN development was scored using a tph-1 ${ }^{\text {prom }}:$. gfp transgene (zdls13). Data are expressed as mean \pm SD and statistical significance was assessed by ANOVA followed by Dunnett's multiple-comparison test. $n>50$, ${ }^{* * *} p<0.0001$. n.s., Not significant. B, C, HSN anatomy of wild-type and dpy-18(ok162) mutant animals. HSN cell bodies migrate to their correct position just posterior to the vulva. However, the HSNL axon crosses over to the right VNC fascicle (white arrowhead) and the HSNL axon extends anteriorly with the HSNR axon (red arrowhead). This left-right bias in axon defects occurs in $94 \%$ of defective animals. Vulval position is marked with a red asterisk. Ventral view, anterior to the left. Scale bar, $20 \mu \mathrm{m}$.

vealed that HSN axon guidance defects of $d p y$-18 mutants are dependent on collagen synthesis and the correct level of ephrin expression. Therefore, we suggest that the molecular environment of $d p y-18$ mutant animals causes aberrant signaling and defective axon guidance at the ventral midline. Together, we have identified a novel function for prolyl 4-hydroxylation in axon guidance regulation a likely conserved mechanism in higher organisms.

\section{Materials and Methods}

Strains. C. elegans strains are listed in the order in which they appear in the figures first and then in tables. The following strains were used:
Figures: RJP133 (Is[tph-1 $\left.{ }^{\text {prom }}:: g f p\right] ;$ RJP1325 dpy-18(ok162); Is[tph-1 $\left.\left.{ }^{\text {prom }}:: g f p\right]\right) ; \mathrm{RJP} 1472$ (phy-2(ok802); Is[tph-1 $\left.\left.{ }^{\text {prom }}:: g f p\right]\right) ;$ RJP154 (phy-3(ok199); Is[tph-1 ${ }^{\text {prom }:: g f p]) ; ~ R J P 1473 ~}$

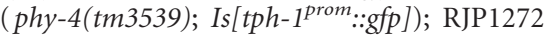

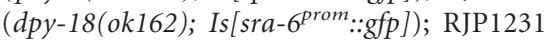

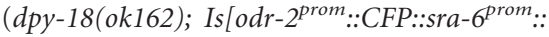
DsRed2]); RJP1476 (dpy-18(ok162); Is[flp$\left.\left.1^{\text {prom }}:: g f p\right]\right)$; RJP882 (rpEx442 Ex[dpy-18prom:: $g f p]) ;$ RJP6 (rpEx1 Ex[WRM0614bC10]; $d p y-$ 18(ok162); Is[tph-1prom::gfp]); RJP7 (rpEx2 Ex[WRM0614bC10]; $d p y-18(o k 162) ; \quad I s[t p h-$ 1prom::gfp]); RJP8 (rpEx3 Ex[WRM0614bC10]; dpy-18(ok162); Is[tph-1prom::gfp]); RJP217 (rpEx59 Ex[dpy-7prom::dpy-18 cDNA]; dpy18(ok162); Is[tph-1prom::gfp]); RJP218 (rpEx60 Ex[dpy-7prom::dpy-18 cDNA]; dpy-18(ok162); Is[tph-1prom::gfp]); RJP433 (rpEx188 Ex[dpy7prom::dpy-18 cDNA]; dpy-18(ok162); Is[tph1prom:::gfp]); RJP442 (rpEx193 Ex[unc-120prom:: $d p y-18 c D N A] ; d p y$-18(ok162); Is[tph-1prom::gfp]); RJP443 (rpEx194 Ex[unc-120prom::dpy-18 cDNA]; dpy-18(ok162); Is[tph-1prom::gfp]); RJP650 (rpEx301 Ex[unc-120prom::dpy-18 cDNA]; dpy18(ok162); Is[tph-1prom:::gfp]); RJP1502 (rpEx662 Ex[unc-129prom:: dpy-18 cDNA]; dpy-18(ok162); Is[tph-1prom::gfp]); RJP1503 (rpEx663 Ex[unc129prom::dpy-18 cDNA]; dpy-18(ok162); Is[tph1prom:::gfp]); RJP1504 (rpEx664 Ex[unc-129prom:: dpy-18 cDNA]; dpy-18(ok162); Is[tph-1prom::gfp]); RJP1505 (rpEx665 Ex[unc-47prom:::dpy-18 cDNA]; dpy-18(ok162); Is[tph-1prom::gfp]); RJP1506 (rpEx666 Ex[unc-47prom::dpy-18 cDNA]; dpy18(ok162); Is[tph-1prom:::gfp]); RJP1507 (rpEx667 Ex[unc-47prom:: dpy-18 cDNA]; dpy-18(ok162); Is[tph-1prom::gfp]); RJP1508 (rpEx668 Ex[punc47::dpy-18cDNA] + [punc-129::dpy-18cDNA]; dpy-18(ok162)); RJP1509 (rpEx669 Ex[punc-47:: $d p y-18 c D N A]+[p u n c-129:: d p y-18 c D N A] ; d p y-$ 18(ok162)); RJP1510 (rpEx670 Ex[punc-47::dpy-18 cDNA] + [punc-129::dpy-18 cDNA]; dpy18(ok162)); RJP851 (emb-9(hc70); Is[tph-1 prom ::gfp]); RJP1666 (emb-9(hc70); dpy-18(ok162); Is[tph-1 $\left.\left.1^{\text {prom }}:: g f p\right]\right) ; \quad$ RJP852 (let-2(b246); Is[tph-1 $\left.\left.{ }^{\text {prom }}:: g f p\right]\right)$; RJP1667 (let-2(b246); dpy18(ok162); Is[tph-1 ${ }^{\text {prom }:: g f p]) ; ~ R J P 1668 ~(c l e-~}$ 1(cg120); Is[tph-1 $\left.\left.{ }^{\text {prom }}:: g f p\right]\right)$; RJP1669 (cle1(cg120); dpy-18(ok162); Is[tph-1 prom ::gfp]);

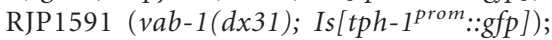
RJP1592 (vab-1(dx31); dpy-18(ok162); Is[tph-1 $\left.\left.1^{\text {prom }}:: g f p\right]\right)$; RJP1595 (vab-2(ju1); Is[tph-1 $\left.\left.{ }^{\text {prom }}:: g f p\right]\right) ; \mathrm{RJP} 1673$ (vab-2(ju1); dpy18(162); Is[tph-1 $\left.\left.{ }^{\text {prom }}:: g f p\right]\right) ; \mathrm{RJP} 1674$ (efn2(ev658); Is[tph-1 $\left.\left.{ }^{\text {prom }}:: g f p\right]\right) ;$ RJP1596 (efn2(ev658); dpy-18(ok162); Is[tph-1 prom ::gfp]); RJP1676 (efn-3(ev696); Is [tph-1 ${ }^{\text {prom }:: g f p]) ; ~}$ RJP1677 (efn-3(ev696); dpy-18(ok162);

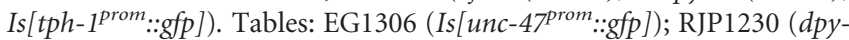

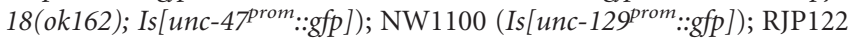
(Is[mec-4 $\left.\left.{ }^{\text {prom }}:: g f p\right]\right)$; RJP1275 (dpy-18(ok162); Is[mec-4 $\left.\left.{ }^{\text {prom }}:: g f p\right]\right)$;

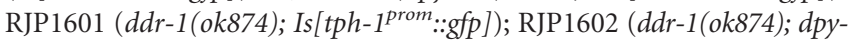
18(ok162); Is[tph-1 $\left.\left.{ }^{\text {prom }}:: g f p\right]\right) ; \mathrm{RJP} 1603$ (nid-1(cg119); Is[tph-1 ${ }^{\text {prom }:: g f p]) ;}$

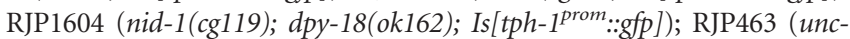

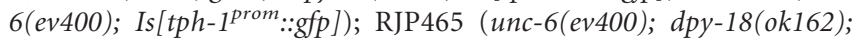

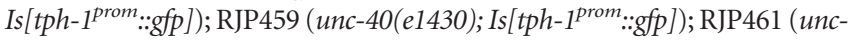

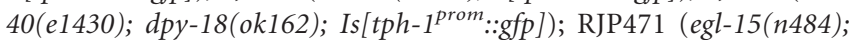

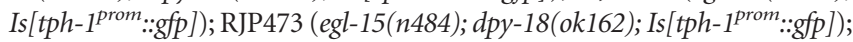

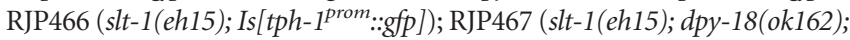

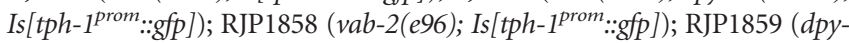


18(ok162); vab-2(e96); Is[tph-1 $\left.\left.1^{\text {prom }}:: g f p\right]\right)$; RJP1860 (vab-1(e2); Is[tph-1 ${ }^{\text {prom }:: g f p]) ; ~ R J P 1861 ~}$ (dpy-18(ok162); vab-1(e2); Is[tph-1 ${ }^{\text {prom }:: g f p]) ;}$ RJP1849 (vab-2(ju1); efn-2(ev658); Is[tph-1 prom ::gfp]); RJP1850 (vab-2(ju1); efn-3(ev696); Is $\left[\right.$ tph-1 $\left.\left.{ }^{\text {prom }}:: g f p\right]\right)$; RJP1599 (efn-2(ev658); efn-3(ev696); Is [tph-1 $\left.\left.1^{\text {prom }}:: g f p\right]\right) ;$ RJP1851 (vab-2(ju1); efn-2(ev658); efn-3(ev696); Is $\left[\right.$ tph-1 $\left.\left.1^{\text {prom }}:: g f p\right]\right) ; \quad$ RJP1852 (rpEx771 Ex[unc-47prom::vab-2 cDNA]; dpy-18(ok162); vab-2(ju1); Is[tph-1prom::gfp]); RJP1853 (rpEx772 Ex[unc-47prom::vab-2 cDNA]; dpy-18(ok162); vab-2(ju1); Is[tph-1prom::gfp]); RJP1854 (rpEx773 Ex[unc-47prom::vab-2 cDNA]; dpy18(ok162); vab-2(ju1); Is[tph-1prom::gfp]); RJP1855 (rpEx774 Ex[unc-129prom::efn-3 cDNA]; dpy-18(ok162); Is[tph-1prom::gfp]); RJP1856 (rpEx775 Ex[unc-129prom::efn-3 cDNA]; dpy-18(ok162); Is[tph-1prom::gfp]); RJP1857 (rpEx776 Ex[unc-129prom::efn-3 cDNA]; dpy-18(ok162); Is[tph-1prom::gfp]). Strains were grown using standard growth conditions on nematode growth medium agar at $20^{\circ} \mathrm{C}$ on Escherichia coli OP50 (Brenner, 1974).

DNA constructs and transgenic lines. Rescue constructs were injected into the $d p y$-18(ok162) mutant background at $5-15 \mathrm{ng} / \mu \mathrm{l}$ with $m y o-2^{\text {prom }}::$ mcherry $(5 \mathrm{ng} / \mu \mathrm{l})$ as injection marker. Expression constructs were injected into $\mathrm{N} 2$ background at $50 \mathrm{ng} / \mu \mathrm{l}$ with myo-2 $2^{\text {prom }:: m c h e r r y ~}(5 \mathrm{ng} / \mu \mathrm{l})$ as injection marker.

Fluorescence microscopy. Animals were grown at $20^{\circ} \mathrm{C}$ and neuroanatomy was scored in $\mathrm{L} 4$ and young adult hermaphrodites by mounting on $5 \%$ agarose on glass slides. Images were taken using an automated fluorescence microscope (AXIO Imager M2, Zeiss) and Zen software (version 3.1, Zen Software).

$q R T-P C R$ assays. RNA was isolated from mixed worm population using standard Trizol-based methods (Chomczynski and Sacchi, 1987). Total cDNA was obtained using TaqMan Reverse Transcription Reagents (Invitrogen, catalog \#N8080234). qRT-PCR reactions were performed in triplicates on a LightCycler 480 System (Roche) using the Maxima SYBR/ ROX qRT-PCR Master Mix (Fermentas, catalog \#K0221) and the results were analyzed using qBase from Biogazelle. Error bars represent the SEM of $\geq 3$ independent sets of samples. Three qRT-PCR reference genes were used.

Electron microscopy. Animals were fixed by high-pressure freezing in $2 \%$ osmium tetroxide, $4 \%$ distilled water in acetone, held at $-90^{\circ} \mathrm{C}$ for $102 \mathrm{~h}$ to begin freeze substitution, then slowly warmed to $0^{\circ} \mathrm{C}$ and rinsed several times in cold $100 \%$ acetone before embedment into Embed812 plastic resin (Hall et al., 2012). Single animals were trimmed by hand and serial thin sections collected onto slot grids, followed by poststaining with uranylacetate for transmission electron microscopy on a Philips CM10 microscope. To detect potential axon crossings between the major and minor fascicles of the VNC, high-power images were collected using a digital camera for every third transverse section proceeding from the anterior preanal ganglion forward along the VNC in several animals.
A

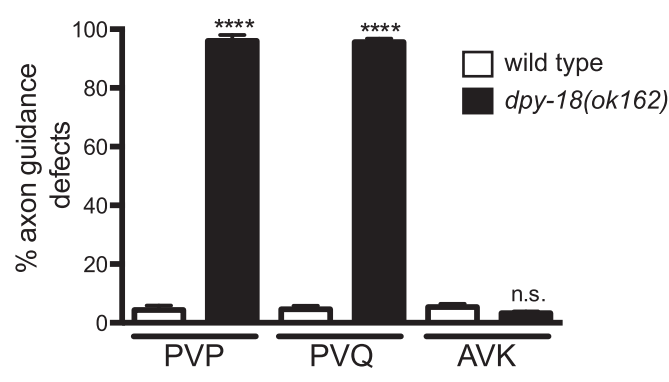

PVPL/R interneurons
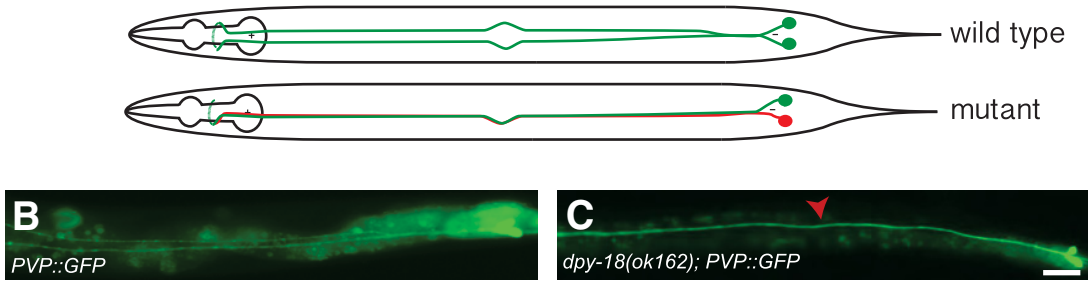

PVQL/R interneurons
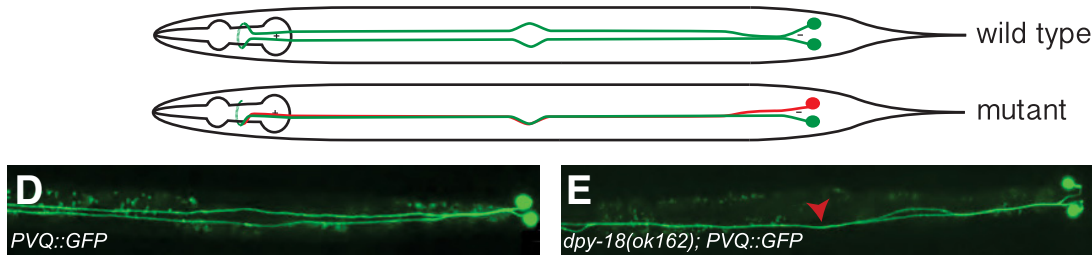
$P V Q \because: G F P$
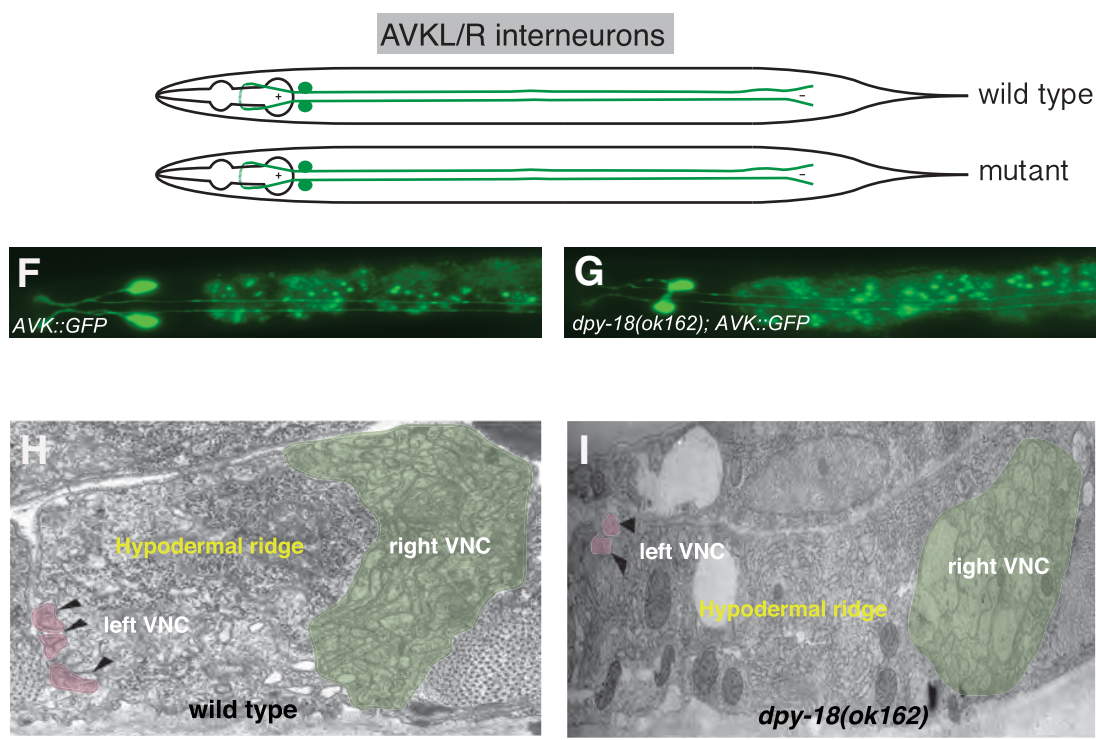

Figure 2. $\quad d p y-18$ regulates posteriorly directed VNC axon guidance. $A$, Quantification of PVP, PVQ, and AVK axonal cross-over defects in $d p y-18(0 k 162)$ mutant animals. Neuroanatomical scoring was performed using the following transgenes: PVP:

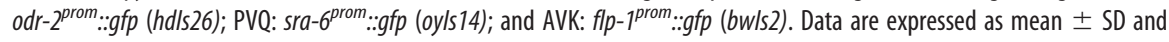
statistical significance was assessed by ANOVA followed by Tukey's multiple-comparison test. $n>50$, ${ }^{* * * *} p<0.0001$. n.s., Not significant. $\boldsymbol{B}-\mathbf{G}$, Neuroanatomy of PVP left and right (PVPL/R), PVQ left and right (PVQL/R), and AVK left and right (AVKL/R) interneurons. Schematic diagrams of these neurons are depicted for wild-type and $d p y-18(0 k 162)$ animals above their respective micrographs. Ventral views, anterior to the left. B, C, PVP anatomy of wild-type and dpy-18(ok162) mutant animals. In dpy18(ok162) mutant animals, the PVP right axon crosses over to the right VNC fascicle (red arrowhead). Scale bar, $20 \mu \mathrm{m} . \boldsymbol{D}, \boldsymbol{E}$, PVQ anatomy of wild-type and $d p y-18(0 k 162)$ mutant animals. In dpy-18(ok162) mutant animals, the PVQ left axon crosses over to the right VNC fascicle (red arrowhead). F, G, AVK anatomy of wild-type and dpy-18(ok162) mutant animals. The AVK axons extend in an anterior to posterior direction. Loss of dpy-18 does not affect the guidance of these axons. $\boldsymbol{H}, \boldsymbol{I}$, Electron micrograph crosssections of wild-type $(\boldsymbol{H})$ and $d p y-18(0 k 162)(\boldsymbol{I})$ mutant animals half way between the vulva and the preanal ganglion. The left VNC of wild type $(\boldsymbol{H})$ contains three axons (PVQL, PVPR, and AVKR) that are deeply embedded in the hypodermal cleft. The left VNC of $d p y-18(0 k 162)$ animals (I) contains two axons that are located in the upper part of the ventral cleft. 
Table 1. Neuronal development in dpy-18(ok162) mutant animals

\begin{tabular}{|c|c|c|c|}
\hline \multirow[b]{2}{*}{ Neurons examined (marker used) } & \multicolumn{2}{|c|}{ Percentage defective animals } & \multirow[b]{2}{*}{$p$ value } \\
\hline & Wild type & $d p y-18(0 k 162)$ & \\
\hline \multicolumn{4}{|l|}{ Interneurons } \\
\hline AVK interneurons (bw/s2) ${ }^{a}$ & 0 & 3 & n.s. \\
\hline PVP interneurons (hdls26) ${ }^{b}$ & 6 & 96 & $* * * *$ \\
\hline PVQ interneurons (oyls 14) & 6 & 94 & $* * * *$ \\
\hline \multicolumn{4}{|l|}{ Motor neurons } \\
\hline \multicolumn{4}{|l|}{ HSN motor neurons (zdls13) ${ }^{d}$} \\
\hline Axon guidance & 8 & 98 & $* * * *$ \\
\hline Cell migration & 5 & 6 & n.s. \\
\hline \multicolumn{4}{|l|}{ Left/right choice ${ }^{e}$} \\
\hline D motor neuron (ox/s12) & 2 & 100 & $* * * *$ \\
\hline DA/DB motor neuron (ev/s82b) & 0 & 13 & ** \\
\hline \multicolumn{4}{|l|}{ Defasciculation $^{f}$} \\
\hline \multicolumn{4}{|l|}{ Dorsal nerve cord } \\
\hline D motor neuron (ox/s12) & 7 & 15 & n.s. \\
\hline DA/DB motor neuron (ev/s82b) & 2 & 34 & $* * * *$ \\
\hline \multicolumn{4}{|l|}{ Ventral nerve cord } \\
\hline D motor neuron (ox/s12) & 4 & 80 & $* * * *$ \\
\hline DA/DB motor neuron (evls82b) & 3 & 14 & ** \\
\hline \multicolumn{4}{|l|}{ Mechanosensory neurons } \\
\hline \multicolumn{4}{|l|}{ Touch cells $(z d / s 4)^{g}$} \\
\hline PLM synapse formation & 4 & 10 & n.s. \\
\hline ALM synapse formation & 0 & 0 & n.s. \\
\hline
\end{tabular}

Neuroanatomical scoring of axon guidance and cell migration in wild-type and dpy-18(0k162) mutant animals. Cell position and axon guidance were assessed using neuron type-specific gfp reporter strains. The position of the neuronal cell bodies and the extension of the axons were compared to control gfp reporter animals. Animals were scored $1 \mathrm{~d}$ after $L 4$ on $\geq 2$ consecutive days. $n>50$, ${ }^{* * *} p<0.0001,{ }^{* *} p<0.002$. n.s., Not significant.

${ }^{a}$ AVK interneurons were scored defective when either AVK right or AVK left axons crossed over to the contralateral side. ${ }^{b}$ PVP interneurons were scored defective when PVP right or PVP left axons crossed over to the contralateral side. 'PVQ interneurons were scored defective when PVQ right or PVQ left axons crossed over to the contralateral side. ${ }^{d} \mathrm{HSN}$ motor neurons were scored defective for either axon cross-over defects or cell migration. Axon guidance was scored as for the interneurons above. No cell migration defects were observed.

${ }^{e}$ Left/right asymmetry was scored as defective when the commissures extended to the inappropriate side.

${ }^{f}$ Fasciculation of the dorsal nerve cord and VNC were scored as defective when $\geq 2$ processes appeared split from one fascicle.

${ }^{9}$ Touch cells: PLMs and ALMs were scored defective when synapses from the PLMs or ALMs failed to extend to the VNC.

Statistical analysis. Statistical analysis was performed in GraphPad Prism 6 using one-way ANOVA for comparison followed by Dunnett's multiplecomparison test or Tukey's multiple-comparison test, where applicable. Values are expressed as mean \pm SD. Differences with a $P$ value $<0.05$ were considered significant.

\section{Results}

\section{$d p y-18$ mutants display VNC axon guidance defects}

Collagen $\mathrm{P} 4 \mathrm{Hs}$ are required for the maturation of collagens and their subsequent assembly into diverse types of extracellular matrices (Myllyharju, 2003). Such matrices also contain a diverse array of signaling molecules, which in combination guide axons to their target sites (Varela-Echavarría and Guthrie, 1997). To ask whether $\mathrm{P} 4 \mathrm{Hs}$ are important for axon guidance, we analyzed knock-out strains on the four $\mathrm{P} 4 \mathrm{H}$ genes in the $C$. elegans genome: $d p y-18 / p h y-1, p h y-2$, phy-3, and phy-4 (Fig. 1; Friedman et al., 2000; Hill et al., 2000; Keskiaho et al., 2008). We focused our analysis on the development the HSNs, which are well studied neurons known to be regulated by multiple conserved molecular pathways and by environmental factors (Desai et al., 1988; Rhiner et al., 2005; Boulin et al., 2006; Pocock and Hobert, 2008; Pedersen et al., 2013). The left and right HSN (HSNL/R) neuronal cell bodies migrate to the midbody region during embryogenesis (Desai et al., 1988). During larval development, HSNL/Rs extend axons in a highly stereotypical manner around the vulval region, before entering the left and right fascicles of the VNC, extending anteriorly and terminating at the nerve ring (Desai et al., 1988).
The HSNL/R axon pairs are separated into the left and right fascicles of the VNC by a midline structure initially made of embryonic motor neuron cell bodies and later by a hypodermal ridge (Boulin et al., 2006). After analyzing the four $\mathrm{P} 4 \mathrm{H}$ genes, we found that loss of $d p y-18$ (using two independent alleles, ok162 and e364) caused VNC axon guidance defects, whereas deletion of the other $\mathrm{P} 4 \mathrm{H}$ enzymes had no detectable effect on axon guidance (Fig. 1; data not shown).

$d p y$-18 mutant animals are short and fat (Dpy) due to defects in cuticular collagen formation (Hill et al., 2000; Myllyharju et al., 2002). We found that in addition to defective body size, loss of $d p y-18$ causes HSNL axons to erroneously extend via the right axon track in $94 \%$ of defective animals, suggesting an asymmetric fault in axon guidance (Fig. 1). To further establish the importance of $d p y$-18 for neuronal development, we analyzed other axon-guidance and cell-migration events in $d p y$ - 18 mutant animals (Fig. 2; Table 1). We found that those axons that navigate the VNC in a posterior-to-anterior direction (HSN, PVP, and PVQ) are severely defective in $d p y$ - 18 mutant animals, whereas the AVK axons, which navigate in the opposite direction, are unaffected by loss of $d p y$-18 (Fig. 2; Table 1). As seen with the HSN neurons (Fig. 1), the vast majority of VNC defects occur where the axon in the left fascicle crosses over to the right. To ask whether the axonal defects observed in $d p y-18$ mutant animals are developmental cross-over events rather than neuronal maintenance flip-over events (Aurelio et al., 2002; Pocock et al., 2008), we analyzed PVQ neurons of freshly hatched L1 larvae. We found that the penetrance of defects was comparable to that of adult animals, suggesting that the defects observed are developmental in nature (data not shown). In addition to the VNC midline defects, we found that the left/right choice of commissural D-type motor neuron axons were defective in $d p y$ - 18 mutant animals, whereas the DA and DB motor neurons were less affected (Table 1). In addition, we found that the longitudinal processes of DA-type, DB-type, and D-type motor neurons exhibit defasciculation defects in $d p y$-18 mutant animals (Table 1; data not shown). Other neurodevelopmental paradigms of cell migration and axon guidance we studied were unaffected by loss of $d p y-18$ (Table 1). Together, our neuroanatomical data indicate that $d p y-18$ plays a major role in the regulation of specific axon guidance events.

\section{Left VNC axons are misplaced in the hypodermal cleft}

To gain a better understanding of why left VNC axons cross over to the contralateral fascicle in $d p y$-18 mutants, we analyzed the VNC by electron microscopy (EM). For technical reasons, we studied electron micrographs in the posterior of L1 larvae (Fig. $2 \mathrm{H}, \mathrm{I}$ ). In wildtype animals, the PVQL, PVPR, and AVKR axons are deeply embedded in the hypodermal cleft (zfr;2Fig. $2 H$ ). In $d p y$-18 mutant animals, however, we found that the left VNC axons are located more toward the top of the hypodermal cleft, or have crossed over to the right VNC, potentially because of altered adhesion in this environment (Fig. 2I). We could not detect any marked changes in the structure of the hypodermal ridge in $d p y$-18 mutant animals in the EM study, suggesting that specific molecules or molecular pathways are dysregulated in $d p y-18$ mutant animals that cause the VNC defects.

\section{$d p y-18$ is expressed in multiple tissues}

Previous studies have shown that $d p y-18$ is predominantly expressed in the hypodermis and in some head and tail neurons (Hill et al., 2000). However, this study did not use the full-length $d p y$-18 promoter to drive expression of fluorescent protein (Hill et al., 2000). To confirm and potentially extend this analysis, we 
A

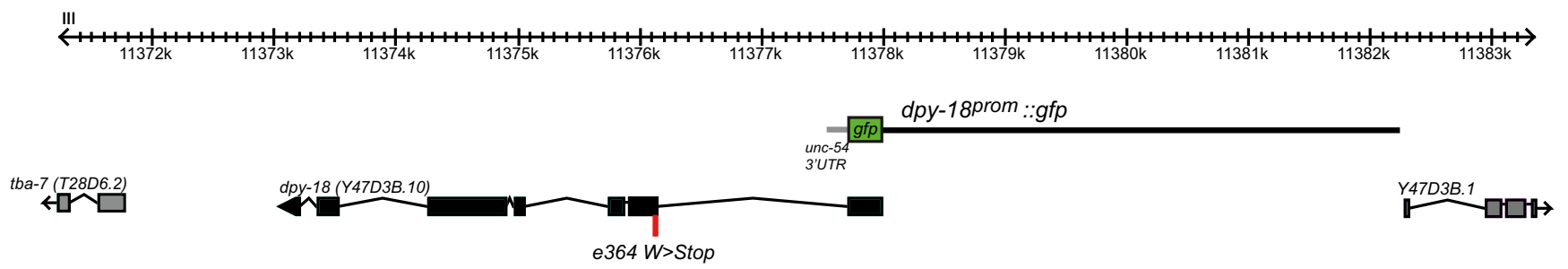

ok162
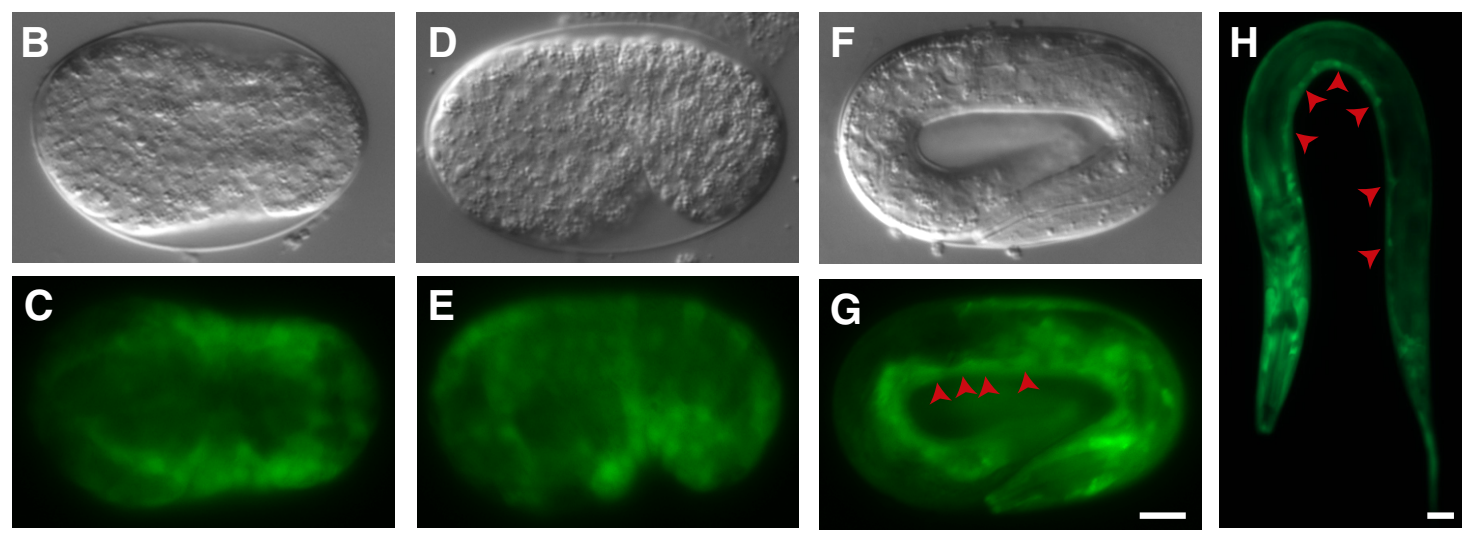

Figure 3. $d p y-18$ is expressed in multiple tissues during development. $A$, dpy-18 locus, mutant alleles, and reporter gene constructs. ok162 and e364 mutant alleles are marked in red. The $d p y-18^{\text {prom }: . g f p}$

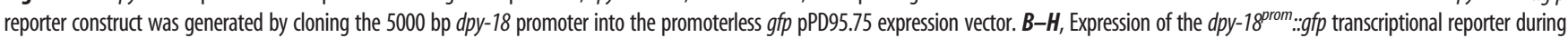
embryonic and larval stages. Expression is first observed at the bean stage $(\boldsymbol{B}-\boldsymbol{E})$ in the hypodermis, muscle, and motor neurons $(\boldsymbol{B}, \boldsymbol{C}$, ventral views; $\boldsymbol{D}, \boldsymbol{E}$ lateral views) and continues to be expressed in these tissues in threefold embryos $(\boldsymbol{F}-\boldsymbol{G}) . \boldsymbol{B}, \boldsymbol{D}, \boldsymbol{F}$, Nomarski micrographs of the embryos. $\boldsymbol{C}, \boldsymbol{E}, \boldsymbol{G}$, Fluorescence images of the same embryos. Anterior to the left. Scale bar, $10 \mu \mathrm{m}$. $\boldsymbol{H}$, In young larvae, dpy-18 $8^{\text {prom }: .: g f p}$ is expressed in the hypodermis, in muscle, in a few unidentified neurons in the head and tail, and in motor neurons. Anterior to the bottom left. Motor neurons are marked by red arrowheads in $\mathbf{G}$ and $\boldsymbol{H}$. Scale bar, $10 \mu \mathrm{m}$.

obtained transgenic strains expressing a full-length transcriptional GFP reporter construct to visualize the expression of $d p y$-18 during development (Fig. 3). Fluorescence in the transcriptional $d p y-18^{p r o m}:: G F P$ reporter strain was first detected at the bean stage of embryogenesis in hypodermis and muscle, and $g f p$ continued to be expressed in these tissues, as well as in motor neurons and in other unidentified neurons, during embryonic development (Fig. 3C, E, G). Postembryonically, $d p y-18^{p r o m}:: G F P$ is expressed in a similar pattern and is more expansive than observed previously (Hill et al., 2000; Fig. $3 H$ ). $d p y$-18 expression is therefore detected at the correct time (bean stage onwards) and in tissues known to be sources of axon guidance regulation along the ventral midline (hypodermis, muscle, neurons).

\section{$d p y-18$ acts in multiple tissues to regulate axon guidance}

As $d p y-18$ is expressed in multiple tissues, we performed transgenic rescue experiments to assess the spatial requirement for $d p y-18$ regulation of axon guidance. To confirm that we could rescue the $d p y$-18(ok162)-induced defects in neuronal development and body size, we transgenically expressed a fosmid containing the entire genomic locus of $d p y-18$ (Fig. $4 A$ ). We found that this fosmid rescued both the Dpy phenotype and HSN axon guidance defects of $d p y$-18 mutant animals (Fig. $4 A$; data not shown). Based on our expression data, which showed that the $d p y$-18 promoter drives expression in multiple tissues, we used a panel of tissue-specific promoters in an attempt to ascertain the $d p y-18$ focus of action in neuronal development. First, we drove $d p y$-18 expression in the hypodermis using the $d p y-7$ promoter. Even though expression of $d p y-18$ in the hypodermis fully rescued the Dpy phenotype, we obtained poor rescue of the axon guidance defects (Fig. 4B; data not shown). This suggests that $d p y-18$ regulates body size and axon guidance via independent mechanisms and/or from different tissues. Indeed, we found that Dpy phenotypes are not a prerequisite of axon guidance phenotypes as mutant worms deficient in the collagens $d p y-7$ and dpy-13 (McMahon et al., 2003) result in a Dpy phenotype but have no detectable defects in axon guidance (data not shown). We next tested whether transgenic expression of $d p y-18$ in other tissues shown to express $d p y-18^{p r o m}:: G F P$ could rescue the HSN axon guidance defects. We drove $d p y$ - $18 \mathrm{cDNA}$ under the control of the unc-120 (body wall muscle), unc-47 (D-type motor neurons), and unc-129 (DA-type and DB-type motor neurons) promoters and found that we could partially rescue the HSN guidance defects in each case. We also used an HSN promoter (egl-6) to drive $d p y$-18 expression and found that this also rescued the $d p y-18$ defects in HSN axon guidance. These data suggest that $d p y-18$ possibly controls HSN axon guidance from multiple tissues, where it could regulate distinct targets in each tissue or the same target from multiple tissues.

\section{$d p y-18$ HSN axonal defects are partially caused by defective collagen}

DPY-18 is required for the correct expression, secretion, and stabilization of the type IV collagens EMB-9 and LET-2 in the BM 


\section{A}

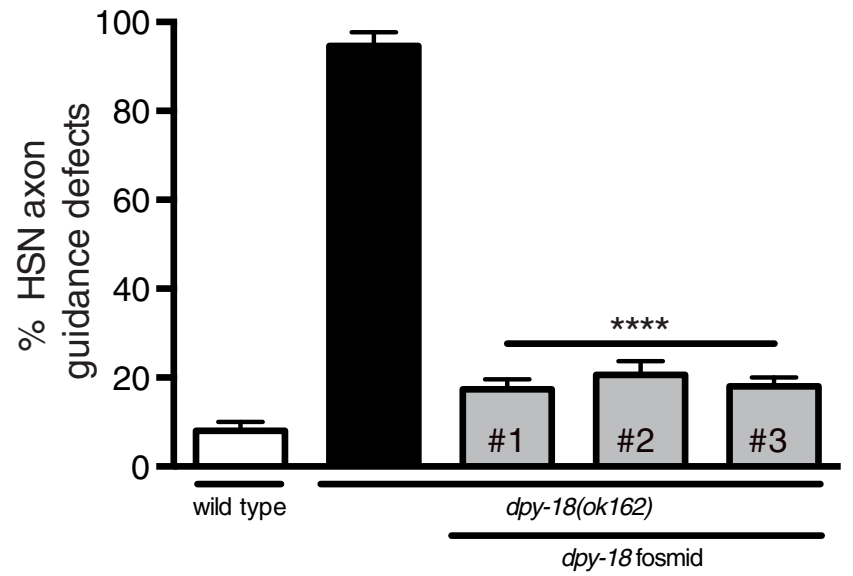

B

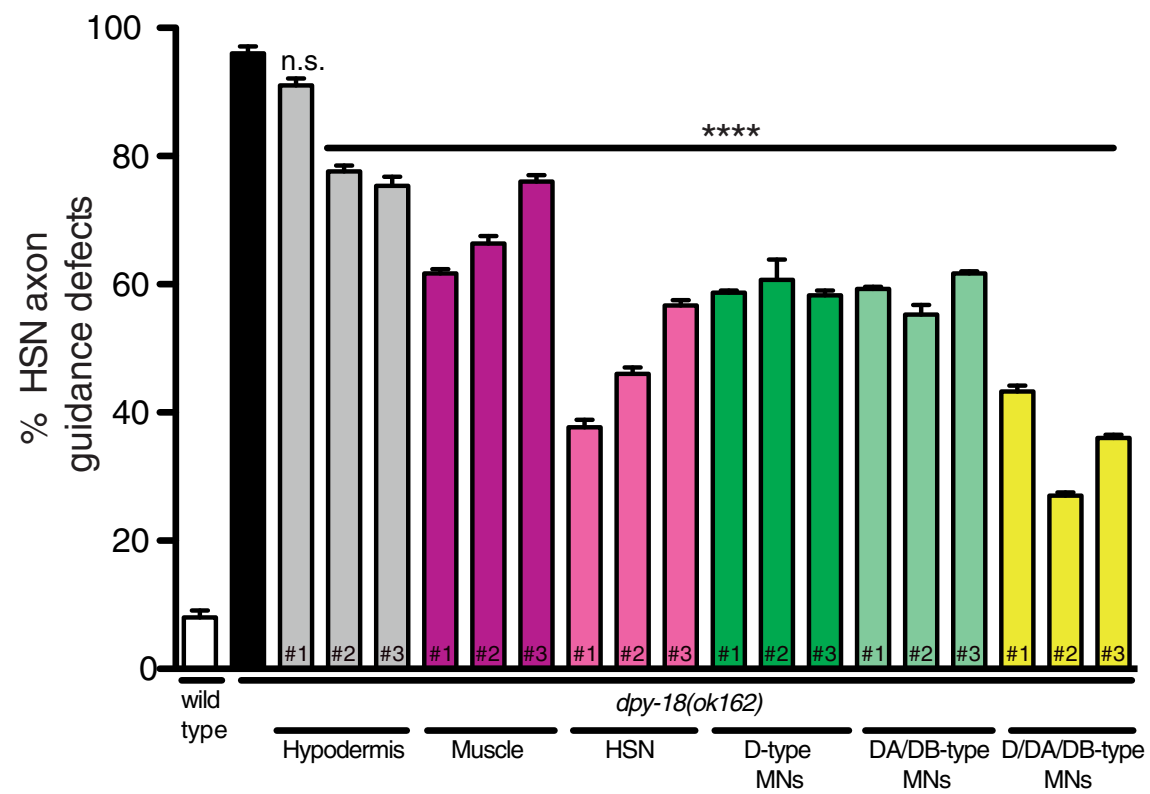

Figure 4. $\quad d p y-18$ is required in multiple tissues to control axon guidance. $\boldsymbol{A}, \mathrm{HSN}$ axon guidance defects of $d p y-18(0 k 162)$ animals are rescued by transgenic expression of a dpy-18-containing fosmid (WRM0614bC10). Data are expressed as mean \pm SD and statistical significance was assessed by ANOVA followed by Dunnett's multiple-comparison test. $n>50,{ }^{* * * *} p<0.0001$. \#, Independent transgenic lines. $\boldsymbol{B}, \mathrm{HSN}$ axon guidance defects of $d p y$-18(ok162) animals are partially rescued by transgenic expression of a dpy-18 CDNA in multiple different tissues. Promoters used for transgenic rescue are as follows: hypodermis, dpy- $7^{\text {prom }}$; muscle unc-120 prom ; HSNs, egl-6 $6^{\text {prom }}$; D-type motor neurons, unc-47 ${ }^{\text {prom }}$; DA/DB-type motor neurons, unc-129 $9^{\text {prom }}$. Data are expressed as mean \pm SD and statistical significance was assessed by ANOVA followed by Dunnett's multiple-comparison test or Tukey's multiple-comparison test, where applicable. $n>50,{ }^{* * *} p<0.0001$. n.s., Not significant; \#, independent transgenic lines.

(Topf and Chiquet-Ehrismann, 2011). Therefore, we tested whether HSN axon guidance is affected in emb-9 and let-2 mutants. As emb-9 and let-2 mutants are lethal, we used the temperature-sensitive alleles emb-9(hc70) and let-2(b246) for this analysis (Guo et al., 1991; Sibley et al., 1994). Both alleles exhibit embryonic lethality at $25^{\circ} \mathrm{C}$, whereas at $20^{\circ} \mathrm{C}$ they are able to grow. We found that at this permissive temperature, HSN axons extend normally in emb-9 and let-2 mutant animals (Table 2). This suggests that either type IV collagens are not required for $\mathrm{HSN}$ axon guidance or that at $20^{\circ} \mathrm{C}$ sufficient type IV collagen is produced to enable guidance. We next combined the emb-9 and let-2 mutant alleles with the $d p y-18$ mutant and studied the effect of reduced type IV collagen production on the HSN guidance phenotype (Table 2). We found the $98 \%$ penetrant defect of $d p y-18$ mutant animals was reduced to 61 and $69 \%$ in the emb-9 and let- 2 mutants respectively at $20^{\circ} \mathrm{C}$. These data suggest that incorrectly folded type IV collagen is deposited on the $\mathrm{BM}$ in $d p y-18$ mutant animals and that this defective collagen is at least partially responsible for the HSN axon guidance defects observed.

The type XV/XVIII collagen CLE-1 has previously been shown to be required for cell migration and axon guidance (Ackley et al., 2001). Therefore, we tested whether defective CLE-1 synthesis is also responsible for the $d p y-18$ mutant defects in HSN axon guidance. cle-1(cg120) mutant animals exhibit weakly penetrant HSN axon guidance defects (20\%). However, we found that loss of cle-1 partially suppresses the $d p y-18$ mutant axonal defects from 98 to $76 \%$ (Fig. 5A; Table 2). Next, we tested whether defective collagens generated in $d p y$ - 18 mutant animals may act through proteins known to bind collagens to direct aberrant axon guidance. We tested DDR-1 and DDR-2 (discoidin domain receptors) and NID-1 (nidogen/entactin), mutants of which cause defects in the nervous system (Kim and Wadsworth, 2000; Unsoeld et al., 2013). We found that loss of $d d r-1$ or nid-1 partially suppressed the HSN axon guidance defects of $d p y-18$ mutant animals (Table 2). Together, these data indicate that $d p y$-18 mutant axon guidance defects are partially dependent on the expression of type IV and type XV/XVIII collagens. Defective deposition of these collagens in the ECM may interfere with the function of DDR-1mediated and NID-1-mediated axon guidance.

\section{$d p y$-18 axonal defects are dependent on ephrin expression}

Our experiments showing that the $d p y-18$ HSN axonal defects are only partially dependent on collagens suggested that modification of other guidance pathways might suppress the highly penetrant $d p y$-18 axon guidance defects. We therefore generated doublemutant combinations between the $d p y$-18(ok162) mutant and loss-of-function mutants of genes that encode conserved axon guidance cues and receptors (Table 3). We found that mutations in netrin, FGF, and Slit/Robo signaling components were unable to suppress the HSN axon guidance defects of $d p y$-18 mutant animals (Table 3). Next, we tested whether the ephrin system plays a role, as it had previously been shown to be important for VNC axon guidance (Boulin et al., 2006; Pocock and Hobert, 2008). First, we found that the VAB-1 Eph receptor acts in a partially kinase-independent manner to direct HSN axon guidance as the kinase-dead allele, $e 2$, exhibits weaker penetrant defects to the $d x 31$-null allele (Fig. 5A). We then assayed ephrin 
Table 2. Double-mutant analysis between dpy-18(ok162) collagen/collageninteracting protein mutants

\begin{tabular}{|c|c|c|c|}
\hline & & HSN guidance defect (\%) & $p$ value \\
\hline dpy-18(ok162) & & 98 & \\
\hline$d d r-1(0 k 874)$ & & 6 & \\
\hline$d d r-1(0 k 874) ; d p y-18(0 k 162)$ & & 78 & $<0.0001$ \\
\hline$d d r-2(0 k 574)$ & & 23 & \\
\hline$d d r-2(0 k 574) ; d p y-18(0 k 162)$ & & 87 & $<0.0001$ \\
\hline nid-1(cg119) & & 70 & \\
\hline nid-1(cg119); dpy-18(ok162) & & 87 & $<0.0001$ \\
\hline cle-1(cg120) & & 20 & \\
\hline cle-1(cg120); dpy-18(ok162) & & 76 & $<0.0001$ \\
\hline emb-9(hc90) & $15^{\circ} \mathrm{C}$ & 6 & \\
\hline emb-9(hc90); dpy-18(ok162) & $15^{\circ} \mathrm{C}$ & 72 & $<0.0001$ \\
\hline emb-9(hc90) & $20^{\circ} \mathrm{C}$ & 14 & \\
\hline emb-9(hc90); dpy-18(ok162) & $20^{\circ} \mathrm{C}$ & 61 & $<0.0001$ \\
\hline let-2(b246) & $15^{\circ} \mathrm{C}$ & 5 & \\
\hline let-2(b246); dpy-18(ok162) & $15^{\circ} \mathrm{C}$ & 95 & n.s. \\
\hline let-2(b246) & $20^{\circ} \mathrm{C}$ & 11 & \\
\hline let-2(b246); dpy-18(ok162) & $20^{\circ} \mathrm{C}$ & 69 & $<0.0001$ \\
\hline
\end{tabular}

VNC defects of HSN axons in dpy-18(ok162) animals are suppressed by mutations in genes encoding type IV and type XV/XVIII collagens (EMB-9, LET-2, and CLE-1) and in collagen-interacting proteins (DDR-1, DDR-2, and NID-1). Data are expressed as mean \pm SD and statistical significance was assessed by ANOVA followed by Dunnett's multiplecomparison test. $n>50,{ }^{* * * *} p<0.0001$. n.S., Not significant.

single-mutant, double-mutant, and triple-mutant combinations, using vab-2-null, efn-2-null, and efn-3-null mutant alleles (ChinSang et al., 1999; Wang et al., 1999), and found that they act in a redundant manner to regulate HSN axon guidance through the Eph receptor (Fig. 5A). We next tested whether reduction of ephrin expression affects the HSN axon guidance defects caused by loss of $d p y-18$. Remarkably, we found that individual loss of the ephrins $v a b-2$, efn-2, and efn-3 suppressed the $d p y-18$ HSN axonal defects from 98 to 29,58 , and $40 \%$ respectively (Fig. $5 B$; Table 3 ). As individual loss of ephrin ligands in wild-type animals causes only mild HSN axonal defects (Fig. 5A), these data suggest that the effect of $d p y-18$ loss on these ephrin ligands may be neomorphic, where they possibly alter signaling of one or more guidance pathways. Indeed, loss of the VAB-1 Ephrin receptor, and as such all canonical ephrin signaling, only mildly suppresses the $d p y-18 \mathrm{HSN}$ axonal defects, indicating that aberrant ephrin signaling is not the major cause of the defects (Fig. $5 B$ ).

Previous work from other systems has shown that the expression level of ephrins can alter their ability to drive axonal trajectories (Kao and Kania, 2011). In addition, collagen is known to regulate the expression level and signaling of multiple axon guidance pathways (Yebra et al., 2003; Wang et al., 2008). We hypothesized that loss of $d p y$-18, possibly through defective collagen formation, may affect ephrin expression. We therefore performed qRT-PCR on $d p y-18(o k 162)$ and $e m b-9(h c 70)$ mutant animals and found that the expression of $v a b-2$, efn-2, and $e f n-3$, but not $v a b$ - 1 , is affected (Fig. $5 C$ ).

We next asked whether the changes in expression of ephrins were causative in the HSN axon guidance defects observed in $d p y-18$ mutant animals. We first focused on $v a b-2$ as expression is increased in $d p y$-18(ok162) mutant animals and loss of $v a b-2$ suppresses the HSN guidance defects of the $d p y-18$ mutant (Fig. $5 B, C)$. We asked whether resupplying $v a b-2$ in motor neurons, the endogenous source of ephrin expression required for midline guidance (Boulin et al., 2006), could reverse the suppression of HSN axon guidance defects observed in $d p y$-18(ok162); vab2(ju1) mutant animals (Fig. 5D). Indeed, we found that transgenic expression of $v a b-2$ in motor neurons restores the highly penetrant $d p y$-18(ok162) mutant phenotype in $d p y-18$ (ok162); $v a b-2(j u 1)$ mutant animals, indicating that induced expression of $v a b-2$ is detrimental to axon guidance in the molecular environment of the $d p y$-18 mutant VNC (Fig. 5D). efn-2 levels are also increased in $d p y$-18(ok162) mutant animals, though not in the emb-9 mutant, suggesting that efn-2 may be responsive to other molecular perturbations than correct assembly of collagen IV (Fig. 5C). However, as with vab-2, loss of efn-2 in $d p y$-18(ok162) mutant animals suppresses the HSN axon guidance defects (Fig. $5 B$ ), indicating that $e f n-2$ upregulation in $d p y$ - 18 mutant animals is detrimental. In contrast to $v a b-2$ and $e f n-2$, the expression of $e f n-3$ is reduced in $d p y-18(o k 162)$ and $e m b-9(h c 70)$ mutant animals, even though loss of $e f n$ - 3 can alleviate $d p y$-18-induced HSN guidance defects (Fig. $5 B, C$ ). Intriguingly, we found that transgenic expression of efn-3 in motor neurons can also reduce the HSN guidance defects of $d p y-18$ (ok162) mutant animals (Fig. $5 E)$. These data suggest that in $d p y-18$ mutant animals, low-level expression of efn-3 is detrimental and either loss or increased expression reverses the adverse effects on axon guidance.

The data above indicate that the ephrin system is responsive to perturbations in the molecular environment controlled by the collagen P4H DPY-18. DPY-18 is required for the correct maturation of EMB-9/collagen IV (Topf and Chiquet-Ehrismann, 2011) and loss of EMB-9 causes dysregulation of the ephrins $v a b-2$ and $e f n-3$ in a similar fashion to loss of DPY-18. Together, our data suggest that loss of $d p y-18$ results in an altered molecular landscape containing defective collagens, and possibly other molecules, that cause dysregulation of ephrin expression and subsequent defective guidance of VNC axons at the midline.

\section{Discussion}

Our study has identified a requirement for proline hydroxylation in neuronal guidance that is supplied by a specific $\mathrm{P} 4 \mathrm{H}$ $\alpha$-subunit, DPY-18 (Fig. 5F). Using tissue-specific rescue experiments, we found that DPY-18 acts from multiple tissues, including VNC motor neurons, to regulate VNC axon guidance. This suggests that $d p y$ - 18 may either act through a variety of pathways to drive axon guidance or that it provides a convivial substratum for axons to navigate through. Interestingly, we observed that the VNC defects in $d p y$-18 mutant animals have a strong left-right bias where left axons cross over to the right fascicle. This leftright bias in neuronal patterning has also been reported recently where loss of Eph signaling causes asymmetric defects in amphid neuron guidance (Grossman et al., 2013). Such findings suggest the asymmetric expression of particular guidance cues and postulate a role for DPY-18 in their localization.

To identify the pathways in which DPY-18 acts, we performed double-mutant analysis and found that reduction of either collagen or ephrin expression alleviated the axon guidance phenotypes caused by DPY-18 loss. In wild-type animals, the type IV collagens EMB-9 and LET-2, together with the type XV/XVIII collagen CLE-1, normally play a minor role in regulating HSN axon guidance (our data; Ackley et al., 2001). In $d p y$-18 mutant animals, however, the presence of defective collagen presumably interferes with collagen-interacting proteins or with other axon guidance signaling pathways to cause aberrant HSN axon guidance. In a similar way, individual loss of any of the ephrins (VAB-2, EFN-2, or EFN-3) weakly affects HSN axon guidance in wild-type animals, yet their dysregulation in the molecular landscape of the $d p y-18$ mutant causes HSN axon guidance defects. We found that the expression levels of the ephrins are altered in mutants of $d p y-18$ and emb-9, suggesting that they are able to respond to perturbations in the extracellular environment, as has been shown after brain injury in other systems (Goldshmit et al., 
A
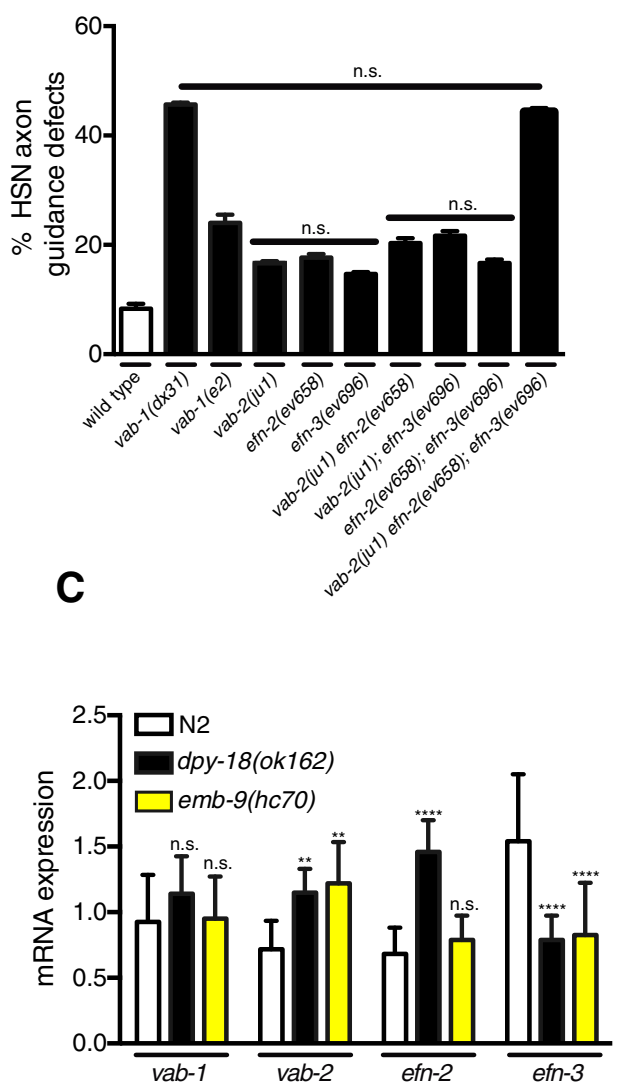

E
B
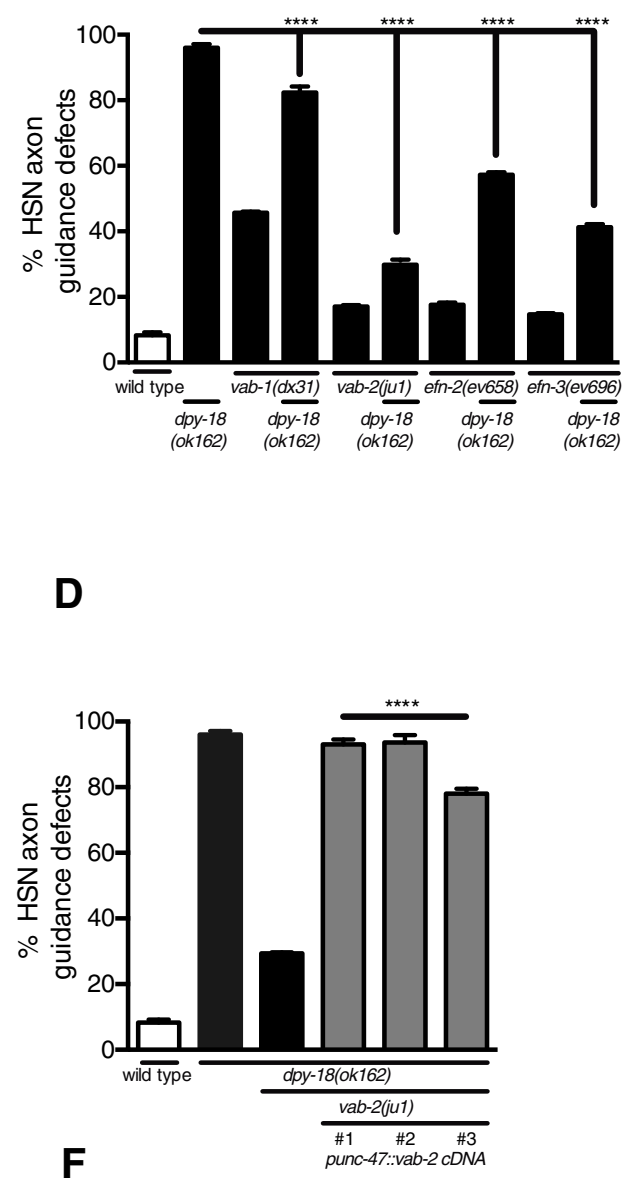

DPY-18

Prolyl 4-hydroxylase

$\downarrow$

EMB-9/LET-2

Collagen IV

$\downarrow$

VAB-2/EFN-2/EFN-3

Ephrin expression

$\downarrow$

Axon midline guidance

Figure 5. Dysregulated ephrin expression causes dpy-18 defects in axon guidance. $A$, Ephrin ligands act redundantly via the VAB-1 Eph receptor to regulate HSN axon guidance. The triple knock-out of the ephrins (vab-2, efn-2, efn-3) phenocopies the vab-1(dx31)-null mutant. Single and double ephrin mutant combinations result in a partially penetrant HSN axon guidance phenotype compared with the vab-1(dx31)-null mutant. The kinase-dead vab-1(e2) allele causes HSN axon guidance defects, though at lower penetrance compared with the vab-1-null mutant, suggesting that VAB-1 plays partially kinase-independent functions in HSN development. Data are expressed as mean \pm SD and statistical significance was assessed by ANOVA followed by Dunnett's multiple-comparison test. $n>50$. n.S., Not significant when comparing single and double ephrin mutant strains and the vab-1 (dx31)-null strain and the ephrin triple mutant. B, HSN axon guidance defects of dpy-18(ok162) animals are partially suppressed by mutations in the ephrin receptor (VAB-1) and the ephrin ligands (VAB-2, EFN-2, and EFN-3). Data are expressed as mean \pm SD and statistical significance was assessed by ANOVA followed by Dunnett's multiple-comparison test. $n>50,{ }^{* * * *} p<0.0001$. Statistical test compares dpy-18(ok162) animals to each double mutant. C, qRT-PCR showing the expression levels of vab-1, vab-2, efn-2, and efn-3 in wild-type, dpy-18(ok162), and emb-9(hc70) mutant animals. ${ }^{* *} p<0.01$, ${ }^{* * * *} p<0.0001$. n.s., Not significant. D, The suppression of $d p y-18(0 k 162)$ HSN axon guidance defects by loss of vab-2 is reversed with transgenic expression of vab-2 CDNA in the D-type motor neurons, under the control of the unc-47 promoter. Expression of the same transgenes in wild-type animals results in a $10 \%$ penetrant defect in HSN axon guidance that is comparable to background levels (data not shown). Data are expressed as mean \pm SD and statistical significance was assessed by ANOVA followed by Dunnett's multiple-comparison test. ${ }^{* * * *} p<0.0001$ when transgenic rescue strains are compared with the $d p y-18(0 k 162)$; vab-2(ju1) double mutant. \#, Independent transgenic lines. $E$, Transgenic expression of efn-3 cDNA in DA and DB motor neurons, under the control of the unc-129 promoter, suppresses the HSN axon guidance defects in $1 p y-18(0 k 162)$ mutant animals. Data are expressed as mean \pm SD and statistical significance was assessed by ANOVA followed by Dunnett's multiple-comparison test. ${ }^{* * *} p<0.0001$ when transgenic rescue strains are compared with the $d p y-18(0 k 162)$. \#, Independent transgenic lines. $F$, Model for the role of the collagen P4H DPY-18 during axon guidance of the C. elegans VNC. DPY-18 is required for faithful maturation and folding of collagen IV (EMB-9/LET-2), which ensures that the ephrins (VAB-2/EFN-2/EFN-3) are correctly expressed via unidentified ECM-mediated signaling pathway(s). 
Table 3. Double-mutant analysis between dpy-18(ok162) and known axon guidance regulators

\begin{tabular}{lrc}
\hline & HSN guidance defects (\%) & $p$ value \\
\hline dpy-18(ok162) & 98 & \\
unc-6(ev400) & 100 & \\
unc-6(ev400); dpy-18(ok162) & 100 & n.s. \\
unc-40(e1430) & 91 & \\
unc-40(e1430); dpy-18(ok162) & 100 & n.s. \\
egl-15(n484) & 24 & \\
egl-15(n484); dpy-18(ok162) & 90 & n.s. \\
vab-1(dx31) & 45 & $<0.0001$ \\
vab-1(dx31); dpy-18(ok162) & 81 & \\
vab-2(ju1) & 16 & $<0.0001$ \\
vab-2(ju1);dpy-18(ok162) & 29 & \\
efn-2(ev658) & 17 & $<0.0001$ \\
efn-2(ev658); dpy-18(ok162) & 58 & \\
efn-3(ev696) & 15 & \\
efn-3(ev696); dpy-18(ok162) & 40 & n.s. \\
sax-3(ky123) & 96 & \\
sax-3(ky123); dpy-18(ok162) & 100 & n.s. \\
slt-1(eh15) & 20 & \\
slt-1(eh15); dpy-18(ok162) & 90 & \\
vic & &
\end{tabular}

VNC defects of HSN axons in $1 p y-18(0 k 162)$ animals are not suppressed by mutations in conserved axon guidance pathways: unc- 6 and unc-40 (netrin); egl-15, clr-1, and let-60 (FGF); slt-1 and sax-3 (Slit/Robo). However, the defects are suppressed by reduced expression of ephrin ligands and the Eph receptor. Data are expressed as mean \pm SD and statistical significance was assessed by ANOVA followed by Dunnett's multiple-comparison test. $n>50$, ${ }^{* * * *} p<0.0001$. n.s., Not significant.

2006). The outcome of ephrin dysregulation in $d p y-18$ mutant animals is VNC axon guidance defects. However, the reason for this ephrin response to ECM perturbations is not understood.

To conclude, our study shows that DPY-18 is required to generate the appropriate molecular landscape to direct VNC axon midline navigation. When $d p y-18$ is removed, axons fail to respect the structural and molecular barriers at the ventral midline, leading to axonal cross-over events. These axonal defects are normally prevented by the correct expression of ephrins that repel axons from the midline (Boulin et al., 2006). In the absence of $d p y-18$ and collagen IV (EMB-9), ephrin expression levels are dysregulated, which may cause disruption of multiple signaling pathways required for axon guidance in the VNC. The prevalence of prolyl 4-hydroxylation in mammalian systems suggests that this modification may also play important roles in neuronal guidance in higher organisms.

\section{References}

Ackley BD, Crew JR, Elamaa H, Pihlajaniemi T, Kuo CJ, Kramer JM (2001) The NC1/endostatin domain of Caenorhabditis elegans type XVIII collagen affects cell migration and axon guidance. J Cell Biol 152:1219-1232. CrossRef Medline

Aurelio O, Hall DH, Hobert O (2002) Immunoglobulin-domain proteins required for maintenance of ventral nerve cord organization. Science 295:686-690. CrossRef Medline

Boulin T, Pocock R, Hobert O (2006) A novel Eph receptor-interacting IgSF protein provides C. elegans motoneurons with midline guidepost function. Curr Biol 16:1871-1883. CrossRef Medline

Brenner S (1974) The genetics of Caenorhabditis elegans. Genetics 77:71-94. Medline

Bülow HE, Boulin T, Hobert O (2004) Differential functions of the C. elegans FGF receptor in axon outgrowth and maintenance of axon position. Neuron 42:367-374. CrossRef Medline

Chin-Sang ID, George SE, Ding M, Moseley SL, Lynch AS, Chisholm AD (1999) The ephrin VAB-2/EFN-1 functions in neuronal signaling to regulate epidermal morphogenesis in C. elegans. Cell 99:781-790. CrossRef Medline

Chomczynski P, Sacchi N (1987) Single-step method of RNA isolation by acid guanidinium thiocyanate-phenol-chloroform extraction. Anal biochem 162:156-159. CrossRef Medline
Desai C, Garriga G, McIntire SL, Horvitz HR (1988) A genetic pathway for the development of the Caenorhabditis elegans HSN motor neurons. Nature 336:638-646. CrossRef Medline

Friedman L, Higgin JJ, Moulder G, Barstead R, Raines RT, Kimble J (2000) Prolyl 4-hydroxylase is required for viability and morphogenesis in Caenorhabditis elegans. Proc Natl Acad Sci U S A 97:4736-4741. CrossRef Medline

Goldshmit Y, McLenachan S, Turnley A (2006) Roles of Eph receptors and ephrins in the normal and damaged adult CNS. Brain Res Rev 52:327345. CrossRef Medline

Grossman EN, Giurumescu CA, Chisholm AD (2013) Mechanisms of Ephrin receptor protein kinase-independent signaling in amphid axon guidance in Caenorhabditis elegans. Genetics 195:899-913. CrossRef Medline

Guo XD, Johnson JJ, Kramer JM (1991) Embryonic lethality caused by mutations in basement membrane collagen of C. elegans. Nature 349:707709. CrossRef Medline

Hall DH, Hartwieg E, Nguyen KC (2012) Modern electron microscopy methods for C. elegans. Methods Cell Biol 107:93-149. CrossRef Medline

Hilario JD, Wang C, Beattie CE (2010) Collagen XIXa1 is crucial for motor axon navigation at intermediate targets. Development 137:4261-4269. CrossRef Medline

Hill KL, Harfe BD, Dobbins CA, L'Hernault SW (2000) dpy-18 encodes an alpha-subunit of prolyl-4-hydroxylase in caenorhabditis elegans. Genetics 155:1139-1148. Medline

Hobert O, Bülow H (2003) Development and maintenance of neuronal architecture at the ventral midline of C. elegans. Curr Opin Neurobiol 13:70-78. CrossRef Medline

Kao TJ, Kania A (2011) Ephrin-mediated cis-attenuation of Eph receptor signaling is essential for spinal motor axon guidance. Neuron 71:76-91. CrossRef Medline

Keskiaho K, Kukkola L, Page AP, Winter AD, Vuoristo J, Sormunen R, Nissi R, Riihimaa P, Myllyharju J (2008) Characterization of a novel Caenorhabditis elegans prolyl 4-hydroxylase with a unique substrate specificity and restricted expression in the pharynx and excretory duct. J Biol Chem 283:10679-10689. CrossRef Medline

Kim S, Wadsworth WG (2000) Positioning of longitudinal nerves in C. elegans by nidogen. Science 288:150-154. CrossRef Medline

McMahon L, Muriel JM, Roberts B, Quinn M, Johnstone IL (2003) Two sets of interacting collagens form functionally distinct substructures within a Caenorhabditis elegans extracellular matrix. Mol Biol Cell 14:1366-1378. CrossRef Medline

Myllyharju J (2003) Prolyl 4-hydroxylases, the key enzymes of collagen biosynthesis. Matrix Biol 22:15-24. CrossRef Medline

Myllyharju J, Kukkola L, Winter AD, Page AP (2002) The exoskeleton collagens in Caenorhabditis elegans are modified by prolyl 4-hydroxylases with unique combinations of subunits. J Biol Chem 277:29187-29196. CrossRef Medline

Paulsson M (1992) Basement membrane proteins: structure, assembly, and cellular interactions. Crit Rev Biochem Mol Biol 27:93-127. CrossRef Medline

Pedersen ME, Snieckute G, Kagias K, Nehammer C, Multhaupt HA, Couchman JR, Pocock R (2013) An epidermal microRNA regulates neuronal migration through control of the cellular glycosylation state. Science 341: 1404-1408. CrossRef Medline

Pocock R, Hobert O (2008) Oxygen levels affect axon guidance and neuronal migration in Caenorhabditis elegans. Nat Neurosci 11:894-900. CrossRef Medline

Pocock R, Bénard CY, Shapiro L, Hobert O (2008) Functional dissection of the C. elegans cell adhesion molecule SAX-7, a homologue of human L1. Mol Cell Neurosci 37:56-68. CrossRef Medline

Rhiner C, Gysi S, Fröhli E, Hengartner MO, Hajnal A (2005) Syndecan regulates cell migration and axon guidance in C. elegans. Development 132: 4621-4633. CrossRef Medline

Schneider VA, Granato M (2006) The myotomal diwanka (lh3) glycosyltransferase and type XVIII collagen are critical for motor growth cone migration. Neuron 50:683-695. CrossRef Medline

Sibley MH, Graham PL, von Mende N, Kramer JM (1994) Mutations in the alpha 2(IV) basement membrane collagen gene of Caenorhabditis elegans produce phenotypes of differing severities. EMBO J 13:3278-3285. Medline 
Steimel A, Wong L, Najarro EH, Ackley BD, Garriga G, Hutter H (2010) The Flamingo ortholog FMI-1 controls pioneer-dependent navigation of follower axons in C. elegans. Development 137:3663-3673. CrossRef Medline

Tessier-Lavigne M, Goodman CS (1996) The molecular biology of axon guidance. Science 274:1123-1133. CrossRef Medline

Topf U, Chiquet-Ehrismann R (2011) Genetic interaction between Caenorhabditis elegans teneurin ten-1 and prolyl 4-hydroxylase phy-1 and their function in collagen IV-mediated basement membrane integrity during late elongation of the embryo. Mol Biol Cell 22:3331-3343. CrossRef Medline

Unsoeld T, Park JO, Hutter H (2013) Discoidin domain receptors guide axons along longitudinal tracts in C. elegans. Dev Biol 374:142-152. CrossRef Medline

Varela-Echavarría A, Guthrie S (1997) Molecules making waves in axon guidance. Genes Dev 11:545-557. CrossRef Medline

Wang X, Roy PJ, Holland SJ, Zhang LW, Culotti JG, Pawson T (1999) Multiple ephrins control cell organization in C. elegans using kinase- dependent and -independent functions of the VAB-1 Eph receptor. Mol Cell 4:903-913. CrossRef Medline

Wang X, Harris RE, Bayston LJ, Ashe HL (2008) Type IV collagens regulate BMP signalling in Drosophila. Nature 455:72-77. CrossRef Medline

White JG, Southgate E, Thomson JN, Brenner S (1976) The structure of the ventral nerve cord of Caenorhabditis elegans. Philos Trans R Soc Lond B Biol Sci 275:327-348. CrossRef Medline

Yebra M, Montgomery AM, Diaferia GR, Kaido T, Silletti S, Perez B, Just ML, Hildbrand S, Hurford R, Florkiewicz E, Tessier-Lavigne M, Cirulli V (2003) Recognition of the neural chemoattractant Netrin-1 by integrins alpha6beta 4 and alpha3beta1 regulates epithelial cell adhesion and migration. Dev Cell 5:695-707. CrossRef Medline

Yu TW, Bargmann CI (2001) Dynamic regulation of axon guidance. Nat Neurosci 4 [Suppl]:1169-1176. Medline

Zallen JA, Yi BA, Bargmann CI (1998) The conserved immunoglobulin superfamily member SAX-3/Robo directs multiple aspects of axon guidance in C. elegans. Cell 92:217-227. CrossRef Medline 